The incidence of chemotherapy-induced peripheral neuropathy (CIPN) in the population of cancer patients is estimated at 3-7\% in cytostatic monotherapy and as high as 38\% in the case of polytherapy. While testing drugs that may reduce the damage to the peripheral nervous system, particular attention should be paid to their protective action against the severe and painful complication in the patient. Another aspect, perhaps a more important one, is the confidence that application of preventive drugs will not exert a significant impact on progression of the neoplastic disease or the effectiveness of the causal treatment. Many drugs have been tested for prevention of CIPN; however, none of them have thus far been irrefutably proven to possess preventive properties. No guidelines on chemotherapy-induced peripheral neuropathy preventive action have been established, either. This article is an attempt to present reports from the available literature about the possibilities of prevention of CIPN.

Key words: chemotherapy-induced peripheral neuropathy, neuropathic pain, side effects, prevention.

\section{Chemotherapy-induced peripheral neuropathy. Part II. Prevention}

\author{
Krzysztof Brzeziński
}

Outpatient Department for Pain Management, Institute of Rural Health, Lublin, Poland

Pain symptoms experienced by patients with a cancer disease usually have a mixed nature, as nociceptive and visceral pain is accompanied by a neuropathic component related to the damage to the peripheral nervous system (PNS). Management of neuropathic pain is extremely difficult; therefore, proper diagnosis and implementation of appropriate treatment contribute to alleviation of the symptoms [1-3].

Damage to a nerve, an anatomical structure, usually produces pain symptoms through external pressure (tumour growth; presence of bone material in pathological fracture; rarely, metastases to nerves). From a neuropathological point of view, the sequence of events includes two steps. The first one is characterized by occurrence of neurogenic pain (caused by nerve compression) which, when prolonged, results in damage to the nervous fibres and, consequently, neuropathic pain.

Another form of neuropathic pain is chemotherapy-induced peripheral neuropathy (CIPN). In this case, axonal damage is induced by the action of cytostatics and therefore the syndrome is classified as toxic neuropathy [4].

The incidence of CIPN in the population of cancer patients is estimated at $1-12 \%$ [5], although recent reports allow a presumption that the prevalence of the phenomenon is increasing [6]. Slightly different conclusions can be drawn from the paper by Cavaletti and Zanna [7], who estimate the incidence at 3-7\% in the case of cytostatic monotherapy and as much as 38\% in polytherapy.

Chemotherapy-induced peripheral neuropathy cannot be regarded as a homogeneous syndrome, given the great variety of cytostatic drugs exhibiting different modes of damaging the PNS. Symptomatology and localization of the symptoms are the only common factors (these issues were addressed in a previous paper [8]). No safe and effective model of management of a CIPN patient has been developed so far. The authors of proposed algorithms concurrently emphasize that evidence-based medicine provides no convincing proof; hence the possibilities of implementation of these algorithms are limited [9].

Initiation of chemotherapy is accompanied by the doctor's awareness that occurrence of the discussed syndrome is highly probable and may even lead to discontinuation of the causal treatment. This paper is an attempt to present reports from current literature concerning the possibilities of prevention of CIPN.

\section{Methods of chemotherapy-induced peripheral neuropathy prevention}

While testing drugs that could possibly decrease the damage to the PNS, attention should be paid to their protective action against severe and painful complications in the patient. Another, perhaps more important, aspect is the confidence that the application of preventive drugs will not exert a significant impact on the progression of the neoplastic disease or on the effectiveness of the causal treatment. Model tests of numerous substances yielded promising results; yet, in clinical trials, either their effectiveness was not proven or they exhibited pro-carcinogenic activity. The results of investigations 
Table 1. List of drugs tested for their effectiveness in CIPN prevention

\begin{tabular}{|c|c|c|c|c|}
\hline \multirow[t]{2}{*}{ Tested drug } & \multirow[t]{2}{*}{ Cytostatic drug } & \multirow[t]{2}{*}{ Limitations } & \multicolumn{2}{|c|}{ Conclusions } \\
\hline & & & PREV (+/-) & $\mathrm{CHEM}(+/-)$ \\
\hline $\mathrm{Ca}^{2+}, \mathrm{Mg}^{2+}$ ions & oxaliplatin & & + & \\
\hline vit. E $600 \mathrm{mg}$ & cisplatin & & + & + \\
\hline amifostine & cisplatin, paclitaxel & & $+/-$ & $+/-$ \\
\hline glutathione & cisplatin & (small group) & + & + \\
\hline glutamine & paclitaxel & & + & $?$ \\
\hline$N$-acetylcysteine & oxaliplatin & & + & $?$ \\
\hline acetyl-L-carnitine & cisplatin, paclitaxel & (no placebo, small group) & + & $?$ \\
\hline carbamazepine & oxaliplatin & & - & $?$ \\
\hline oxcarbazepine & oxaliplatin & (no placebo, small group) & + & $?$ \\
\hline venlafaxine & oxaliplatin & (no placebo, small group) & + & $?$ \\
\hline
\end{tabular}

PREV - effectiveness of prevention, CHEM - impact of the drug on chemotherapeutic activity, $(+)$ - positive, $(-)$ - negative

of some methods of PNS damage prevention will be discussed below; Table 1 contains the cumulative data.

\section{Calcium and magnesium ions}

Gamelin et al. presented encouraging results of a study aiming at prevention of PNS damage by application of magnesium and calcium ions during oxaliplatin therapy [10]. The positive results of these observations were even more interesting since this management would not involve high expenses or require specialized equipment. Hochster et al. [11] aimed to verify this hypothesis, yet the investigation had to be discontinued due to accelerated neoplastic disease progression. Although the positive action of magnesium and calcium in CIPN prevention was confirmed, the ions were concurrently reported to reduce the anti-tumour activity of oxaliplatin. This phenomenon implies that application of ions of some metals prevents both neuro-and cytotoxicity [12]. Unfortunately, the current knowledge of this activity does not promote such management due to the risk of reduced effectiveness of chemotherapy.

\section{Vitamin E}

In 2003, Pace et al. published the results of investigations employing the anti-oxidative activity of vitamin $\mathrm{E}$ in the dose of $300 \mathrm{mg} /$ day [13]. An important observation was the lack of interaction of vitamin $E$ with the anti-tumour action of cisplatin. The studies were repeated by Argyriou et al. [14-16], who reported very positive effects of application of vitamin $E$ in the dose of $600 \mathrm{mg} /$ day both in combination with cisplatin and paclitaxel therapies. Therefore, supplementation with vitamin $\mathrm{E}$ may be regarded as a method of CIPN prevention, although further investigations are advisable [12, 17].

\section{Amifostine}

Amifostine is an organic thiophosphate compound, whose anti-oxidative activity is based on scavenging free rad- icals. The first attempts to use this compound for prevention of CIPN demonstrated that the pathology of the peripheral nervous system occurred at a higher cumulative dose of cisplatin with no concurrent side effects [18]. Subsequent tests performed to determine the effectiveness of this procedure in patients treated with cisplatin combined with paclitaxel [19] and cisplatin with cyclophosphamide [20] did not reveal any positive effect. In turn, the study conducted by Kemp et al. demonstrated substantial limitations in the use of this compound due to the frequent events of hypotonia during amifostine infusion [20].

\section{Glutathione}

The preventive effect of glutathione involves reduction of the cisplatin concentration in spinal ganglion cells and an impact on cisplatin-induced protein p53 [21, 22]. However, there is a risk that glutathione will reduce the anti-tumour activity due to increased renal excretion of cisplatin. Although randomized placebo-controlled trials have confirmed the effectiveness of CIPN prevention without a substantial impact on the basic treatment [23], positive results were obtained from a small number of patients tested; therefore this procedure cannot be fully recommended.

\section{Glutamine}

Glutamine is a specific endogenous amino acid which should be regarded as an exogenous amino acid in cell damage. Its role in CIPN prevention involves inhibition of oxidative stress [24]. It was reported that this amino acid may have an effect on production of the nerve growth factor (NGF), whose presence may be an important inducer of CIPN [25, 26]. Supplementation with non-essential amino acids may be related to the risk of their impact on the development of all fast-dividing cells; therefore, it is possible (although not proved clinically) that glutamine may induce tumour expansion. Clinical trials have proved the efficacy of this amino acid in prevention of CIPN in patients treated with paclitaxel; yet, 
the fear of reduced effectiveness of its anticancer activity limits implementation of this procedure [17, 24].

\section{$\mathrm{N}$-acetylcysteine}

$\mathrm{N}$-acetylcysteine plays a role in protecting the cells of the nervous system by increasing glutathione levels in the spinal ganglion cells. Only one study has reported the positive effect of this preventive procedure in patients treated with oxaliplatin [27]; the insufficient number of patients tested does not allow realistic assessment of the safety of application of the drug.

\section{Acetyl-L-carnitine}

The preventive mechanism of this compound is not known, but it was found that it both exerts an effect on regeneration of damaged nerve fibres and reduces pain impulses in fibres A and C [28-30]. Non-placebo-controlled trials performed on a small group of patients treated with paclitaxel or cisplatin have demonstrated positive effects [31]; nevertheless, they are insufficient to recommend the drug for clinical use.

\section{Human recombinant interleukin}

Cytokines may play a substantial role in induction of the damage to the PNS. Model studies demonstrated a preventive action of interleukin 6 in CIPN induced by paclitaxel, cisplatin, and vincristine [32]. Further clinical trials did not confirm the assumed preventive activity, as there were no significant differences between the patients receiving therapy and those receiving placebo [33-35].

\section{Antiepileptic drugs}

Impairment of sodium channel function is one of the probable mechanisms responsible for neuronal damage accompanying oxaliplatin therapy [36-38]. Since carbamazepine inhibits the activity of these channels, attempts were made to apply the drug to prevent CIPN. Unfortunately, no differences were found between the test and placebo groups [36].

Oxcarbazepine is a more recent anti-epileptic drug that affects both sodium and calcium channels. Although trials performed to assess its preventive action during oxaliplatin therapy yielded a positive outcome, they were non-placebocontrolled and involved a small group of patients. Therefore, they should be regarded as pilot studies providing no recommendations for using the drug in clinical practice [36]. Conclusions from the reports of the use of venlafaxine reveal similar limitations [39].

The effectiveness in prevention of CIPN has been tested in many drugs with no conclusive outcome. No guidelines for preventive procedures applied during chemotherapy have been established, either. Some hope can be attached to the few studies reporting attempts to implement CIPN management algorithms [40], which may alter the current approach to prevention and management of this severe pain syndrome. There is a need for further prospective studies evaluating the effectiveness of CIPN prevention and its safety associated with absence of any impact on the causal treatment of cancer.

\section{References}

1. Chong MS, Bajwa ZH. Diagnosis and treatment of neuropathic pain. J Pain Symptom Manage 2003; 25: S4-S11.

2. Allen RR. Neuropathic pain in the cancer patient. Neurol Clin 1998; 16: 869-88.

3. Dieleman JP, Kerklaan J, Huygen FJ, Bouma PA, Sturkenboom MC. Incidence rates and treatment of neuropathic pain conditions in the general population. Pain 2008; 137: 681-8.

4. Umapathi T, Chaudhry V. Toxic neuropathy. Curr Opin Neurol 2005; 18: 574-80.

5. Paice JA. Mechanisms and management of neuropathic pain in cancer. J Support Oncol 2003; 1: 107-20.

6. Lavoie Smith EM, Bakitas MA, Homel P, Fadul C, Meyer L, Skalla K, Bookbinder M. Using quality improvement methodology to improve neuropathic pain screening and assessment in patients with cancer. J Cancer Educ 2009; 24: 135-40.

7. Cavaletti G, Zanna C. Current status and future prospects for the treatment of chemotherapy-induced peripheral neurotoxicity. Eur J Cancer 2002; 38: 1832-7.

8. Brzeziński K. Chemotherapy-induced polyneuropathy. Part I. Pathophysiology. Wspolczesna Onkol 2012; 16: 72-8.

9. Visovsky C, Collins M, Abbott L, Aschenbrenner J, Hart C. Putting evidence into practice: evidence-based interventions for chemotherapyinduced peripheral neuropathy. Clin J Oncol Nurs 2007; 11: 901-13.

10. Gamelin L, Boisdron-Celle M, Delva R, Guérin-Meyer V, Ifrah N, Morel A, Gamelin E. Prevention of oxaliplatin-related neurotoxicity by calcium and magnesium infusions: a retrospective study of 161 patients receiving oxaliplatin combined with 5 -fluorouracil and leucovorin for advanced colorectal cancer. Clin Cancer Res 2004; 10: 4055-61.

11. Hochster HS, Grothey A, Childs BH. Use of calcium and magnesium salts to reduce oxaliplatin-related neurotoxicity. J Clin Oncol 2007; 25: 4028-9.

12. Albers JW, Chaudhry V, Cavaletti G, Donehower RC. Interventions for preventing neuropathy caused by cisplatin and related compounds. Cochrane Database Syst Rev 2007; (1): CD005228.

13. Pace A, Savarese A, Picardo M, et al. Neuroprotective effect of vitamin $\mathrm{E}$ supplementation in patients treated with cisplatin chemotherapy. J Clin Oncol 2003; 21: 927-31.

14. Argyriou AA, Chroni E, Koutras A, Ellul J, Papapetropoulos S, Katsoulas G, Iconomou G, Kalofonos HP. Vitamin E for prophylaxis against chemotherapy-induced neuropathy. Neurology 2005; 64: 26-31.

15. Argyriou AA, Chroni E, Koutras A, Iconomou G, Papapetropoulos S, Polychronopoulos P, Kalofonos HP. Preventing paclitaxel-induced peripheral neuropathy: a phase II trial of vitamin E supplementation. J Pain Symptom Manage 2006; 32: 237-44.

16. Argyriou AA, Chroni E, Koutras A, Iconomou G, Papapetropoulos S, Polychronopoulos P, Kalofonos HP. A randomized controlled trial evaluating the efficacy and safety of vitamin E supplementation for protection against cisplatin-induced peripheral neuropathy: final results. Support Care Cancer 2006; 14: 1134-40.

17. Kaley TJ, Deangelis LM. Therapy of chemotherapy-induced peripheral neuropathy. Br J Haematol 2009; 145: 3-14.

18. Mollman JE, Glover DJ, Hogan WM, Furman RE. Cisplatin neuropathy. Risk factors, prognosis, and protection by WR-2721. Cancer 1988; 61: 2192-5.

19. Moore DH, Donnelly J, McGuire WP, Almadrones L, Cella DF, Herzog TJ, Waggoner SE; Gynecologic Oncology Group. Limited access trial using amifostine for protection against cisplatin- and three-hour paclitaxel-induced neurotoxicity: a phase II study of the Gynecologic Oncology Group. J Clin Oncol 2003; 21: 4207-13.

20. Kemp G, Rose P, Lurain J, et al. Amifostine pretreatment for protection against cyclophosphamide-induced and cisplatin-induced toxicities: results of a randomized control trial in patients with advanced ovarian cancer. J Clin Oncol 1996; 14: 2101-12.

21. Cascinu S, Cordella L, Del Ferro E, Fronzoni M, Catalano G. Neuroprotective effect of reduced glutathione on cisplatinbased chemotherapy in advanced gastric cancer: a randomized doubleblind placebo-controlled trial. J Clin Oncol 1995; 13: 26-32. 
22. Park SA, Choi KS, Bang JH, Huh K, Kim SU. Cisplatin-induced apoptotic cell death in mouse hybrid neurons is blocked by antioxidants through suppression of cisplatin-mediated accumulation of $\mathrm{p} 53$ but not of Fas/Fas ligand. J Neurochem 2000; 75: 946-53.

23. Cascinu S, Catalano V, Cordella L, et al. Neuroprotective effect of reduced glutathione on oxaliplatin-based chemotherapy in advanced colorectal cancer: a randomized, double-blind, placebocontrolled trial. J Clin Oncol 2002; 20: 3478-83.

24. Savarese DM, Savy G, Vahdat L, Wischmeyer PE, Corey B. Prevention of chemotherapy and radiation toxicity with glutamine. Cancer Treat Rev 2003; 29: 501-13.

25. Vahdat L, Papadopoulos K, Lange D. Reduction of paclitaxelinduced peripheral neuropathy with glutamine. Clin Cancer Res 2001 7: 1192-7.

26. De Santis S, Pace A, Bove L, et al. Patients treated with antitumor drugs displaying neurological deficits are characterized by a low circulating level of nerve growth factor. Clin Cancer Res 2000; 6: 90-5.

27. Lin PC, Lee MY, Wang WS, et al. N-acetylcysteine has neuroprotective effects against oxaliplatin-based adjuvant chemotherapy in colon cancer patients: preliminary data. Support Care Cancer 2006; 14: 484-7.

28. Pisano C, Pratesi G, Laccabue D, et al. Paclitaxel and cisplatin-induced neurotoxicity: a protective role of acetyl-L-carnitine. Clin Cancer Res 2003; 9: 5756-67.

29. Flatters SJ, Xiao WH, Bennett GJ. Acetyl-L-carnitine prevents and reduces paclitaxel-induced painful peripheral neuropathy. Neurosci Lett 2006; 397: 219-23

30. Xiao WH, Bennett GJ. Chemotherapy-evoked neuropathic pain: abnormal spontaneous discharge in A-fiber and C-fiber primary afferent neurons and its suppression by acetyl-L-carnitine. Pain 2008; 135: 262-70.

31. Bianchi G, Vitali G, Caraceni A, Ravaglia S, Capri G, Cundari S, Zanna C, Gianni L. Symptomatic and neurophysiological responses of paclitaxel- or cisplatin-induced neuropathy to oral acetyl-Lcarnitine. Eur J Cancer 2005; 41: 1746-50.

32. Callizot N, Andriambeloson E, Glass J, Revel M, Ferro P, Cirillo R, Vitte PA, Dreano M. Interleukin-6 protects against paclitaxel, cisplatin and vincristine-induced neuropathies without impairing chemotherapeutic activity. Cancer Chemother Pharmacol 2008; 62: 995-1007.

33. Davis ID, Kiers L, MacGregor L, et al. A randomized, double-blind ed, placebo-controlled phase II trial of recombinant human leukemia inhibitory factor (rhuLIF, emfilermin, AM424) to prevent chemotherapy-induced peripheral neuropathy (see comment). Clin Cancer Res 2005; 11: 1890-8.

34. van den Bent MJ. Prevention of chemotherapy-induced neuropathy: leukemia inhibitory factor (comment). Clin Cancer Res 2005; 11: 1691-3.

35. Wen PY. A randomized, double-blinded, placebo-controlled phase II trial of recombinant human leukemia inhibitory factor (rhuLIF, emfilermin, AM424) to prevent chemotherapy-induced peripheral neuropathy (comment). Clin Cancer Res 2005; 11: 1685-6.

36. Argyriou AA, Chroni E, Polychronopoulos P, et al. Efficacy of oxcarbazepine for prophylaxis against cumulative oxaliplatin-induced neuropathy. Neurology 2006; 67: 2253-5.

37. Argyriou AA, Polychronopoulos P, Iconomou G, Chroni E, Kalofonos HP. A review on oxaliplatin-induced peripheral nerve damage. Cancer Treat Rev 2008; 34: 368-77.

38. von Delius S, Eckel F, Wagenpfeil S, et al. Carbamazepine for prevention of oxaliplatin-related neurotoxicity in patients with advanced colorectal cancer: final results of a randomised, controlled, multicenter phase II study. Invest New Drugs 2007; 25: 173-80.

39. Durand JP, Brezault C, Goldwasser F. Protection against oxaliplatin acute neurosensory toxicity by venlafaxine. Anticancer Drugs 2003; 14: 423-5.

40. Smith EM, Bakitas MA, Homel P, Piehl M, Kingman L, Fadul CE, Book binder M. Preliminary assessment of a neuropathic pain treatment and referral algorithm for patients with cancer. J Pain Symptom Manage 2011; 42: 822-38.

\section{Address for correspondence}

\section{Krzysztof Brzeziński}

Outpatient Department for Pain Management Institute of Rural Health

Jaczewskiego 2

20-950 Lublin, Poland

e-mail: k.brzezinski@op.pl

Submitted: $\quad 14.01 .2012$

Accepted: $\quad$ 15.02.2012 